\title{
Validity of Quantitative Ultrasound and Bioelectrical Impedance Analysis against Dual X-Ray Absorptiometry for Measuring Bone Quality and Body Composition in Children ${ }^{\dagger}$
}

\author{
Maryam Delshad 1, Kathryn L. Beck 1, Cathryn A. Conlon 1, Owen Mugridge 1, \\ Marlena C. Kruger ${ }^{2}$ and Pamela R. von Hurst ${ }^{1, *}$ \\ 1 School of Sport, Exercise and Nutrition, College of Health, Massey University, Auckland 0632, \\ New Zealand; M.DelshadSiyahkaly@massey.ac.nz (M.D.); k.1.beck@massey.ac.nz (K.L.B.); \\ c.conlon@massey.ac.nz (C.A.C.); o.mugridge@massey.ac.nz (O.M.) \\ 2 School of Health Sciences, College of Health, Massey University, Palmerston North 4474, New Zealand; \\ m.c.kruger@massey.ac.nz \\ * Correspondence: P.R.vonHurst@massey.ac.nz \\ + Presented at the 2018 Nutrition Society of New Zealand Annual Conference, Auckland, New Zealand, \\ 28-30 November 2018.
}

Published: 7 March 2019

Background: Dual energy X-ray absorptiometry (DXA) is a well-regarded device for primarily measuring bone mineral density (BMD) and body composition. However, its use is limited in children since it is expensive, time-consuming, lacks portability, and exposes children to ionizing radiation. The objective was to examine the validity of quantitative ultrasound (QUS) and bioelectrical impedance analysis (BIA) measurements against DXA for bone quality and body composition in children (8-13 years) living in Auckland, New Zealand.

Methods: Whole body bone mineral content (BMC), BMD, and body composition were measured with DXA (QDR Discovery A, Hologic, USA), BMD and calcaneal stiffness index (SI) with QUS (Sahara QUS, Hologic, USA), and BIA measurements on the InBody 230 (Biospace Ltd., Seoul, Korea). Relative validity was assessed using Pearson correlation coefficients, cross-classification, and weighted K-statistic

Results: Healthy children ( $n=127,58$ boys) were recruited. Positive correlations between QUS -SI and DXA (BMC and BMD) were observed (range $=0.40-0.45)(p<0.05)$. QUS-SI correctly classified $>50 \%$ of participants into the correct tertile and $<10 \%$ into the opposite tertile when compared with DXA-BMD. Moderate agreement $(\mathrm{K}=0.4)$ was found through weighted $\mathrm{k}$-statistic analysis (between QUS-SI and DXA-BMD). Correlations existed between BIA and DXA for lean mass, fat mass and percentage body fat (range $=0.8-0.97)(p<0.01)$. Cross-classification showed a range of $70 \%-84 \%$ of participants were correctly categorized into the same tertile. Weighted K-statistic illustrated good agreement $(\mathrm{K}=0.6-0.8)$ between BIA and DXA variables.

Conclusion: We found that the calcaneal QUS-SI appears to be a valid method for identifying children with low BMD as identified by DXA, and BIA is a valid method to assess children's body composition status since there was a good relative agreement between BIA parameters and DXA values. Our results suggest that calcaneal QUS and BIA could be used to investigate bone health and body composition among children, respectively.

Supplementary Materials: The presentation is available online at www.mdpi.com/2504-3900/8/1/16/s1.

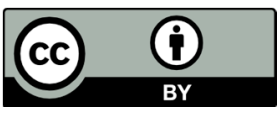

(C) 2019 by the authors. Licensee MDPI, Basel, Switzerland. This article is an open access article distributed under the terms and conditions of the Creative Commons Attribution (CC BY) license (http://creativecommons.org/licenses/by/4.0/). 